\title{
Errors Induced by the Atmosphere in Microwave Range Measurements
}

\author{
H. B. Janes and M. C. Thompson, Jr. \\ Contribution From the Central Radio Propagation Laboratory, National Bureau of Standards, Boulder, Colo.
}

(Received March 3, 1963; revised May 19, 1964)

\begin{abstract}
This paper describes experimental measurements designed to study atmosphere-induced errors in microwave baseline tracking systems. The ground-to-air configuration was simulated by 300 to $400 \mathrm{~m}$ baselines on level ground east of Boulder, Colo., and a fixed target antenna on a mountain top at a range of about $15 \mathrm{~km}$ and a path elevation angle of 44 milliradians. A radiofrequency of $9.4 \mathrm{Gc} / \mathrm{s}$ was used. Continuous recordings were made of variations in apparent range, range difference, and refractive index. The data are analyzed in terms of power spectra. The correlation between range and surface refractivity variations and the correlation of range variations on a djacent paths are discussed.
\end{abstract}

\section{Introduction}

The accuracy of distance measurements made by observing the transit time (or phase) of radio signals transmitted over the distance in question is affected by space and time variations of the radio refractive index of the atmosphere. The National Bureau of Standards has conducted an extensive theoretical and experimental program to study these refractive index variations, their effect on radio distance measurements, and the extent to which distance measurement errors can be reduced by appropriate corrections based on refractive index measurements [Thompson and Janes, 1959; Thompson, Janes, and Kirkpatrick, 1960; Thompson, Janes, and Freethey, 1960]. In this experiment, certain features of an or thogonal baseline tracking system were simulated in order to isolate and study the errors contributed by the lower atmosphere in tracking an elevated target.

\section{Experimental Site}

The criteria used in site selection were: (1) The target should be located on a mountain with terrain dropping off sharply in the direction of the baselines and as high as possible, consistent with all-weather accessibility. (2) The baselines should be located on nearly level ground at an altitude low relative to the target. They should, of course, command an unobstructed view of the target with no obvious sources of anomalous multipath effects. The baseline site, referred to in this report as the Boulder Creek Site, fulfilled these requirements reasonably well.

1 This study was sponsored by the Air Force Missile Test Center, U.S. Air Force Systems Command, under Contract No. A F08(606)-3776 with the General Electric Company.
Figure 1 shows the baseline arrangement. In this report, the $380 \mathrm{~m}$ baseline, nearly perpendicular to the propagation path, will be referred to as the normal baseline and the $520 \mathrm{~m}$ baseline, which is more nearly parallel to the propagation path, will be called the "parallel" baseline. (The latter actually forms an angle of approximately $30^{\circ}$ with the path.)

The target site is located near the summit of Green Mountain, west of the city of Boulder, at an altitude $2,240 \mathrm{~m}$ above sea level and at a horizontal distance of about $15.5 \mathrm{~km}$ from the central station at Boulder Creek. The angle of elevation from the central station to the target is approximately 44 milliradians. A profile of the propagation path is shown in figure 2 .

Parabolic antennas were used throughout. The target antenna was $46 \mathrm{~cm}$ in diameter, while the antennas at Boulder Creek were all $137 \mathrm{~cm}$ in diameter, with their centers $130 \mathrm{~cm}$ above ground.

\section{Data Output}

Data were recorded during three periods, each approximately 2 to 3 days in length. These periods were April 4 to 7, 1961; May 9 to 12, 1961; and January 3 to 5,1962 . The discussion that follows will be restricted to the January run.

The raw data consist of continuous recordings of: (1) variations in apparent range at 9,400 Mcs; (2) variations in apparent range difference on both the normal and "parallel" baselines; (3) variations in atmospheric refractivity as monitored by standard wet-and-dry bulb thermometers and barometers and by microwave refractometer cavities about $1.5 \mathrm{~m}$ above ground near the central antenna at Boulder Creek and at Green Mountain. The range and range difference measurement system used is similar to that described previously [Thompson and Vetter, 1958]. 


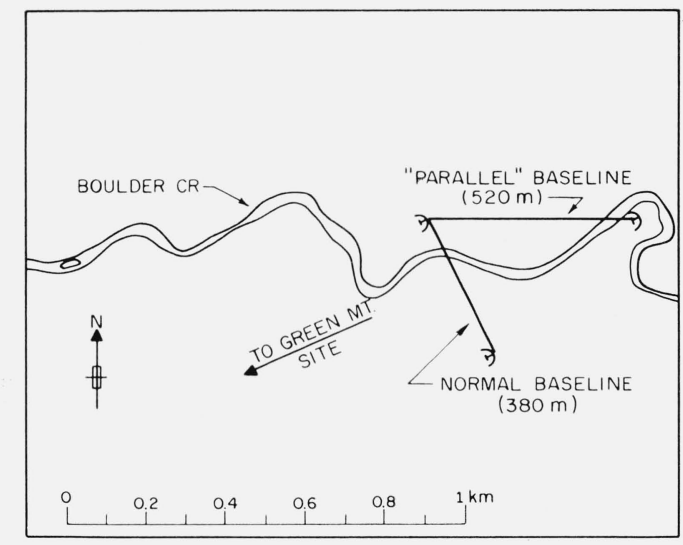

FiguRE 1. Boulder Creek site.

Some sample recordings of range, range difference and refractivity are shown in figure 3. These samples were taken during a relatively "quiet" period from the standpoint of fluctuation intensity. The data include "quiet" periods, "noisy" periods characterized by comparatively large and rapid variations, and periods during which the range data are dominated by large "trends" (i.e., variations

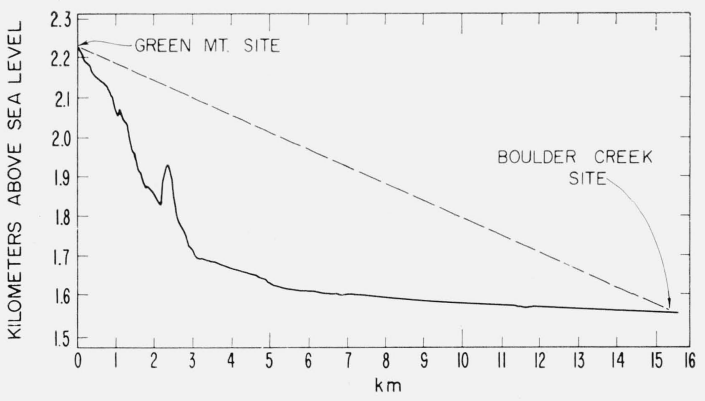

Figure 2. Profile of Boulder Creek-Green Mt. Path.

having periods that are long compared to the sample length). In general, any 2 -day recording period can be expected to vield all three kinds of data. The statistics obtained from 15 minute samples of each of these categories will, in general, differ from each other more than the 2-day means of these statistics will vary from one run to another. In fact, the variability of these short-term statistics is often greater than the difference between mean statistics obtained from Colorado and Florida data.
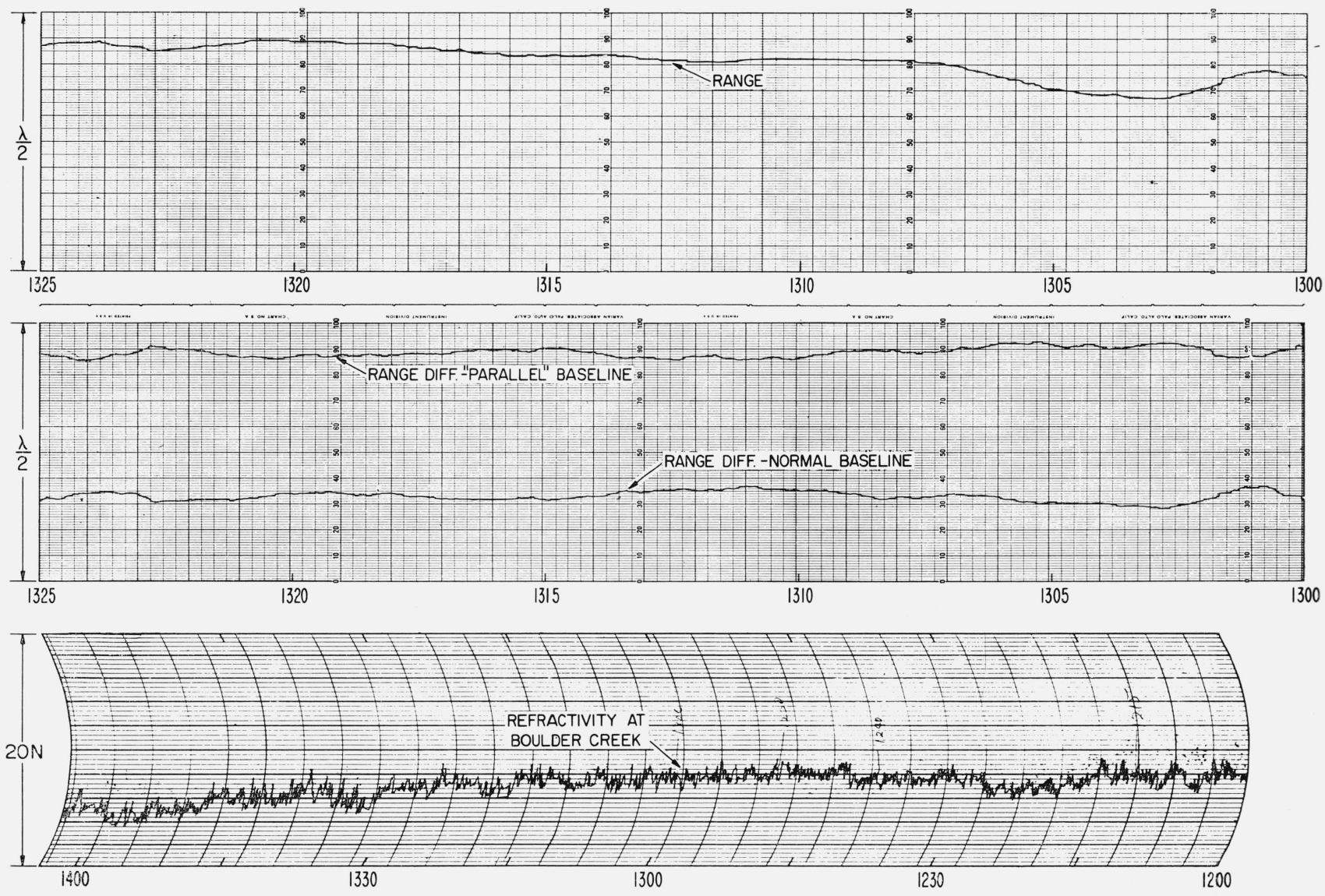

FIGURE 3. Range, range differences and refractivity data showing quiet period in range, January 3,1962 (note differing time scales). 


\section{Data Analysis}

Hour-to-hour variations in range, range difference, and surface refractive index recorded during the January run are shown in figures 4 and 5 . In general, the variations did not follow a clear-cut diurnal cyclic pattern. The largest changes or trends in range and refractivity appear to follow longer term changes in weather.

It is apparent from these graphs and the data samples in figure 3 that the statistical characteristics of the range, range difference, and refractivity fluctuations are quite complicated and do not lend themselves to description in terms of simple statistics such as the variance or standard deviation. Each variable contains a hierarchy of fluctuations, ranging from relatively large, slowly-varying components to a smaller and rapidly varying "noise." Consequently, the variance of any given sample depends not only on the atmospheric conditions prevailing at the time, but also on the pass-band of the recording circuit and the length of the sample.

The power density spectrum (usually referred to as simply the "power spectrum") provides a useful description of such data. It not only takes into account the particular recording pass band and sample length, but also permits estimation of the variance that would have been obtained with any of a wide range of other choices of pass band and sample length.

In the discussion that follows, the power spectrum analysis of the range, range difference, and refractive index data will be used to illustrate some important correlations among these variables.

\section{Correlation of Apparent Range and Surface Refractive Index}

The primary consideration in estimating the rms range error (after correcting for refractive index variations) is the correlation between the apparent range variations, $R(t)$, and the variations in surface refractivity, $N(t)$. The nature of this correlation should, therefore, be examined before going further.

In a homogeneous atmosphere (i.e., one in which $N$ has the same value throughout the volume of interest but varies with time), variations in $R(t)$ would be perfectly correlated with variations in $N$ observed at any point. That is:

$$
R(t)=R_{0}\left[1+N(t) \times 10^{-6}\right]
$$

where $R_{0}$ is the true distance. In fact, this simplified picture is inaccurate because of random time variations in $N$ which are not well correlated throughout the volume, and by more or less persistent changes in $N$ with altitude (the vertical $N$ profile). The correlation between $N$ at one point and $R$ has been found to be a function of the data pass band involved. In other words, when the data samples are long enough to include the relatively large, low-frequency variations, $N$ at either path terminal is relatively well correlated with $R$. For example, the long-term variations in $N$ and $R$ shown in figure 4 show con-

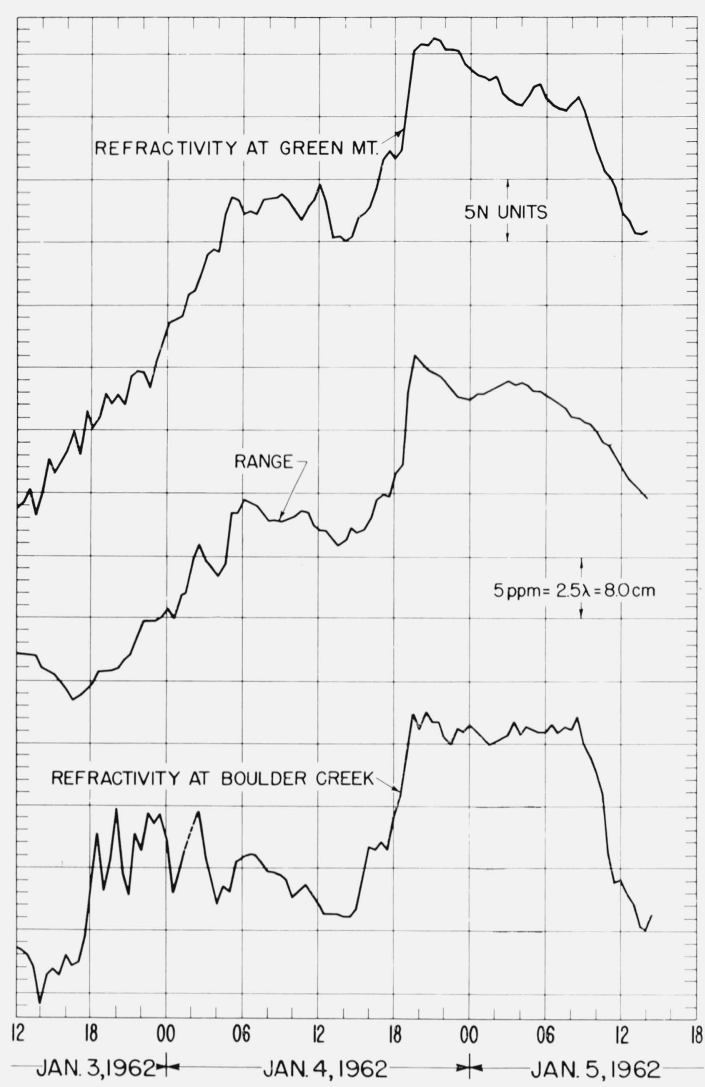

Figure 4. Range and refractivity variations, January 3-5, 1962.
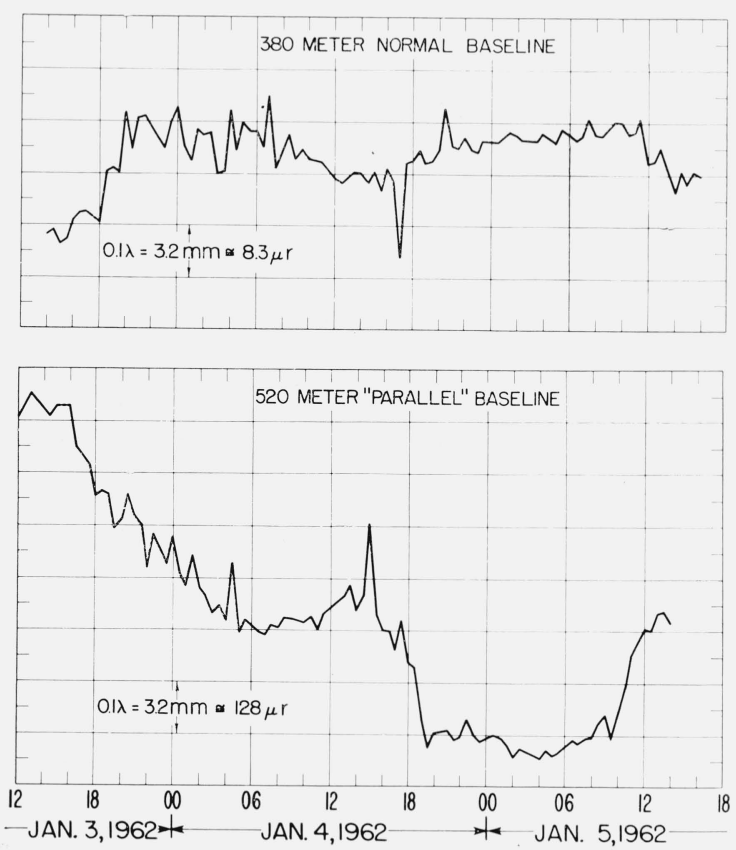

Figure 5. Range difference variations, January 3-5, 1962. 


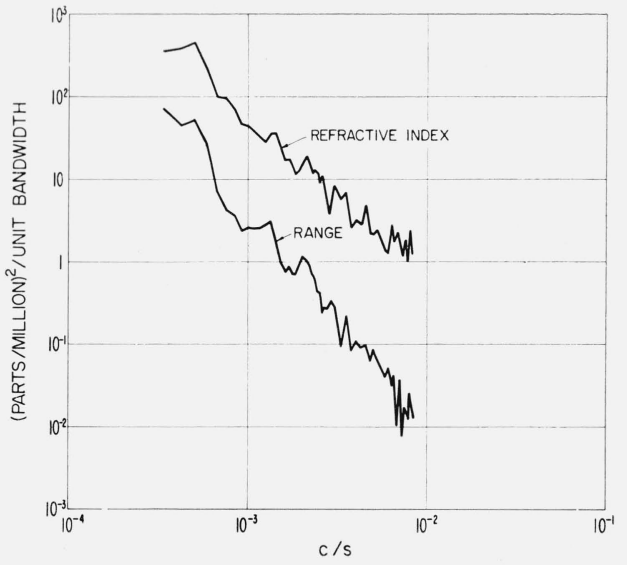

Figure 6. Power spectra of range and surface refractive index variations, January 3-5, 1962.

siderable correlation, but short segments of these records show no significant correlation (see, for example, the $N$ and $R$ data samples in fig. 3 ).

It should also be noted that when both $N$ and $R$ variations are expressed in terms of parts per million of their nominal values (as in fig. 4), their long-term variations are approximately equal in magnitude, but the short-term (e.g., second-to-second) variations in $N$ are, in general, large compared to the corresponding $R$ variations.

To describe the situation quantitatively, figure 6 shows the power spectra of $N$ and $R$. In the frequency range shown, the spectral density of $N$ is roughly proportional to $f^{-1.5}$ and the spectral density of $R$ is proportional to about $f^{-2.5}$. These agree quite well with previous measurements [Thompson, Janes, and Kirkpatrick, 1960]. The two spectra diverge toward the high frequency end, and it appears that with extrapolation toward lower frequencies they would coincide somewhere in the vicinity of $10^{-4} \mathrm{c} / \mathrm{s}$. The similarity of the long-term $N$ and $R$ data such as that shown in figure 4 indicates that the two spectra would coincide at all lower frequencies.

This coincidence and the divergence at higher frequencies are consistent with a hypothetical atmosphere in which time variations in $N$ appearing at a given spectral frequency are caused by spatial structure components having dimension or scale inversely proportional to the given spectral frequency. In other words, the slow $N$ variations (e.g., diurnal variations) are the result of atmospheric changes occurring almost simultaneously over relatively large areas, while the higher frequency fluctuations are caused by small turbulent eddies. In this picture, the two spectra coincide at frequencies sufficiently low so that the associated spatial scales are large compared to the path length. In this spectral region, $N$ variations at any two points on the path are highly correlated, and changes in $R$, which is the line average of $N$ along the path, will be essentially equal to changes in $N$ observed at any point. However, at higher frequencies, $N$ variations are not correlated at all points on the path; i.e., it would be possible to find at least two points at which the $N$ variations are independent random variables. Since in this hypothetical atmosphere the spatial scales are inversely proportional to the spectral frequency, the number of effectively independent random $N$ variables is directly proportional to frequency. Recalling from elementary statistics the fact that the variance of the average of $q$ independent random variables (having equal variances) is inversely proportional to $q$, one would expect the following relationship between the variance spectral densities of $N$ and $R$ :

$$
W_{R}(f) \cong \frac{1}{q(f)} W_{N}(f),
$$

where $W_{R}(f)$ and $W_{N}(f)$ are the spectral densities of $R$ and $N$, respectively, and $q(f)$ is the maximum number of points on the path at which the variations in $N$ in the neighborhood of spectral frequency $f$ are essentially independent.

Let $l(f)$ be the spatial scale, defined as the minimum distance at which variations in $N$ in the neighborhood of $f$ are uncorrelated. Under the hypothesis, for a given path length, $L$, we have

$$
q(f)=\left\{\begin{array}{l}
\frac{L}{l(f)} \text { for } l(f)<L \\
1 \text { for } l(f) \geq L .
\end{array}\right.
$$

If we define $f_{0}$ such that $l\left(f_{0}\right)=L$ (and since $l(f)$ $\left.\propto \frac{1}{f}\right)$, then

and

$$
q(f)=\frac{l\left(f_{0}\right)}{l(f)}=\frac{f}{f_{0}}, \quad l(f)<L,
$$

$$
W_{R}(f)=\left\{\begin{array}{lr}
\frac{f_{0}}{f} W_{N}(f), & f>f_{0} \\
W_{N}(f), & f \leq f_{0} .
\end{array}\right.
$$

The spectra in figure 6 show the difference in slope indicated by these equations, and it appears that $f_{0}$ is somewhat less than $10^{-4} \mathrm{c} / \mathrm{s}$.

The frequency dependence of the correlation of $N$ and $R$ is also illustrated in figure 7 for the January run. Here the correlation coefficient is plotted versus the lower cutoff frequency of a digital high-pass filtering process. The latter process consists of simply replacing the original data with their deviations from running averages of $n$ data points spaced $\delta$ seconds apart, and then repeating the operation on the deviations themselves to further sharpen the response of the "filter." The nominal cutoff frequency referred to in figure 7 is the $3 \mathrm{~dB}$ point, or $0.86 / \delta n \mathrm{c} / \mathrm{s}$. (The point plotted at $3.6 \times 10^{-6} \mathrm{c} / \mathrm{s}$ is the correlation coefficient of the raw data with no 
high-pass filtering except the filtering effect of the finite sample size itself.) From this curve as well as the power spectra, it is evident that $N$ and $R$ are correlated on this path at fluctuation frequencies ranging from 0 to somewhat below $10^{-4} \mathrm{c} / \mathrm{s}$. Hence it would appear advisable to smooth the refractive index data to remove the uncorrelated higher frequencies before using it to correct range data.

The range error spectrum can be extended toward zero frequency by use of a very low-frequencyspectrum of refractive index variations computed from 8 years of U.S. Weather Bureau data for Denver. This spectrum is shown in figure 8 (along with similar spectra from Florida and Hawaii, to be discussed later). It covers the region from about $10^{-8}$ to $2 \times 10^{-5} \mathrm{c} / \mathrm{s}$, or about 1 cycle per year to 2 cycles per day, and includes the portion of the range variance that can, in principle, be largely eliminated by correcting the range data with surface refractive index data.

\section{Comparison of Range and Range Difference Power Spectra}

In general, the variance $\sigma_{\Delta R}^{2}$ of the apparent range difference variations will be related to the variance $\sigma_{R}^{2}$ of range variations on either of the two paths by the following formula:

$$
\sigma_{\Delta R}^{2}=2 \sigma_{R}^{2}(1-r)
$$

where $r$ is the correlation coefficient between the two single-path range variables. On closely spaced paths (such as the ones discussed here), $r$ is very nearly unity for samples sufficiently long to include the relatively large, hour-to-hour variations, but approaches zero for samples a few minutes in length. In other words, as in the case of range versus refractive discussed in the previous section, this correlation is highest at the low fluctuation frequencies and effectively vanishes at the high frequency end of the power spectrum.

This dependence of correlation on fluctuation frequency is due to the fact that, in general, relatively rapid range fluctuations are caused by the movement of relatively small atmospheric "blobs" across the paths, while slower fluctuations could be caused by blobs large enough to effect both paths simultaneously. In the latter case, the range variations over the two paths will be correlated and the range difference variance will be reduced accordingly.

This is illustrated in figure 9, which shows the range and range difference spectra for the January run. At the high frequency end of the spectra (above about 0.005 to $0.01 \mathrm{c} / \mathrm{s}$ ), the range difference spectral densities on both baselines are about twice as large as the range spectra, indicating that in this region the range variations as seen at each end of either baseline are uncorrelated. At lower frequencies, the range difference spectra are reduced relative to the range spectra, indicating an increasing correlation in range variations with decreasing frequency. If we represent the spectra with a function

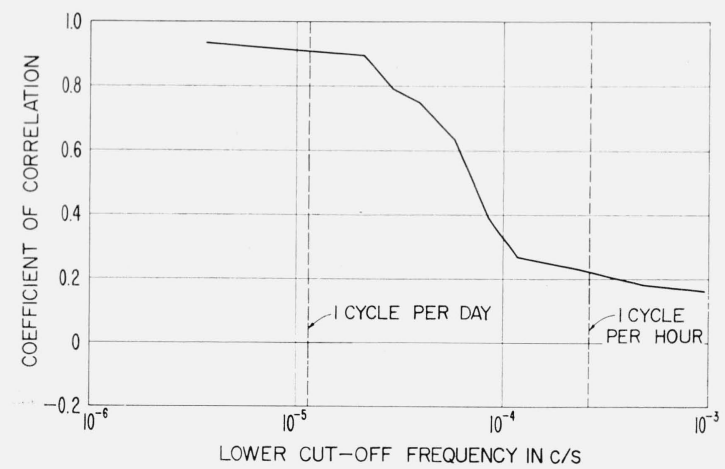

Figure 7. Correlation of range and surface refractive index as a function of high-pass filter cutoff frequency, January 3-5, 1962.

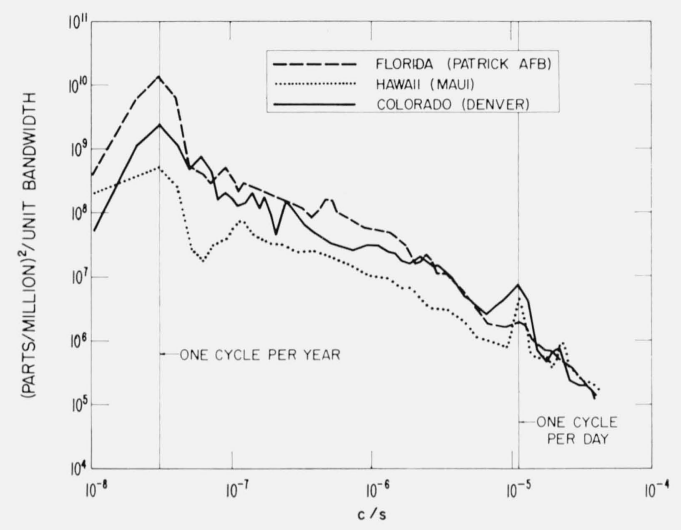

FIgURE 8. Power spectra of long-term refractive index variation. Each spectrum based on approximately 8 years of U.S. Weather Bureau data.

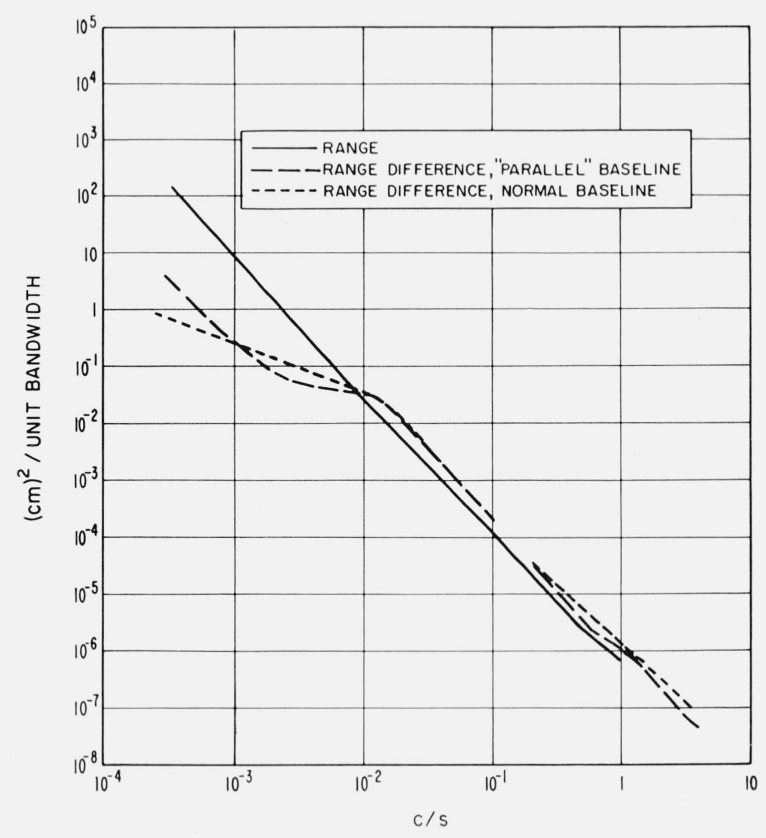

Figure 9. Power spectra of range and range difference variations, January 3-5, 1962. 
of the form

$$
W(f) \propto f^{s},
$$

the average values of $s$ (or the slope of a straightline fit to the spectra on a log-log grid) are -2.4 for the range spectra and for the high frequency end of the range difference spectra. The average slopes of the lower frequency end of the range difference spectra are -1.1 and -0.8 for the "parallel" and normal baselines, respectively.

It has been predicted [Barton, 1963] that, under the assumption of a constant mean wind moving a "frozen" atmosphere across the two paths, the range difference spectrum will be related to the range spectrum by the following:

$$
\begin{aligned}
W_{\Delta R}(f) & =W_{R}(f) G_{B}(f), \\
G_{b}(f) & =2\left(\frac{f}{f_{b}}\right)^{2}, \quad f<f_{b} \\
= & 2, \quad f>f_{b},
\end{aligned}
$$

where $f_{b}$ is proportional to the mean cross-wind speed and inversely proportional to the baseline length, $b$.

Thus the range difference spectral slope at frequencies below $f_{b}$ should be greater by 2 than the range spectral slope. That this prediction is not borne out by the data may be caused by the wide variability of the wind velocity during any 2 or 3 day period which would cause variations in $f_{b}$ and hence a less pronounced difference in the slope of the range and range difference spectra.

\section{Applications and Extensions}

\subsection{Dependence of Range Spectrum on Path Length}

The dependence of range spectral density on the path length can be estimated from an extension of the discussion in section 5 . If $f_{0}$ is taken as the frequency corresponding to time required for a spatial component of dimension $L$ to move across the path then we can assume that

$$
f_{0}=\frac{k}{L},
$$

where $k$ would represent the mean wind speed. With this modification, (4) becomes

$$
\begin{aligned}
W_{R}(f) & =\frac{k}{L f} W_{N}(f) \quad f>f_{0} \\
& =W_{N}(f) \quad f \leq f_{0} .
\end{aligned}
$$

Thus for paths in a region where $W_{N}$ is sensibly homogeneous, the range spectral density (expressed in ppm of the range) is independent of path length for the lower frequencies and inversely proportional to length for the higher frequencies. However, on a slant path such as the one used in this experiment, lengthening the path (but holding the elevation angle constant) might not change $f_{0}$ appreciably, since the path would be extended towards the upper troposphere where the fluctuations in $N$ would be expected to be smaller than those at lower altitudes. Conversely, increasing the elevation angle will have the effect of shortening the portion of the path lying in the troposphere and might increase $f_{0}$ somewhat.

\subsection{Velocity Variations}

The atmospheric errors in the rate of change of range and range difference (i.e., velocity) can be estimated from these experimental data, with some important reservations discussed below. The velocity error contributed by the troposphere in the case of a target with a true angular velocity of zero can be estimated from the spectra of the time derivatives (i.e., rate spectra) of the range and range difference data. The latter are related to the range and range difference spectra by the following:

$$
\begin{aligned}
W_{\dot{R}}(f) & =(2 \pi f)^{2} W_{R}(f) \\
W_{\Delta \dot{R}}(f) & =(2 \pi f)^{2} W_{\Delta R}(f) .
\end{aligned}
$$

The extension of these results to the case of a target with nonzero angular velocity may involve some questionable assumptions. In the latter situation the propagation path is being rotated about its lower terminal at an angular velocity which depends upon the range and velocity of the target. The effect of sweeping the radio beam across the atmosphere is to change the rate of fluctuations. Attempts to estimate moving-path velocity errors [Barton, 1963] from fixed-path data usually involve ascribing a mean normal wind velocity component to the fixed-path data and relating this velocity to an effective tangential path velocity. This process suffers from two basic uncertainties. First, since the wind structure is neither homogeneous nor statistically stationary, the assumption of an average value of wind velocity applicable to the entire path would seem to be unrealistic. Second, when the path is being rotated about its lower terminal to track a moving target, it is not clear what effective tangential velocity should be used to characterize the path movement.

\subsection{Angular Position Variations as a Function of Baseline Length}

The rms angular position variations for longer baselines can be estimated from the data by appropriate modification of the range difference power spectra. It was pointed out previously that, at sufficiently low fluctuation frequencies, the range variations on the two paths will be correlated because the atmospheric blobs responsible for the variations are large enough to extend across both paths simultaneously. This correlation shows up as a reduction in range difference spectral density (relative to the range spectral density) at low frequencies. 
From the spectra in figure 9, it appears that this reduction occurred below about 0.005 to $0.01 \mathrm{c} / \mathrm{s}$.

For a given wind structure, increasing the path separation (i.e., baseline length) should reduce the frequency below which the variations are correlated. In fact, this "cutoff" frequency should be inversely proportional to baseline length.

\subsection{Extension of Results to Other Climates}

With regard to the application of these data to other climatic regions, there is evidence ['Thompson and Janes, 1964; Thompson, Janes, and Kirkpatrick, 1960 ] that the variance contribution from the high frequency end of the spectrum (i.e., above about $0.01 \mathrm{c} / \mathrm{s}$ ) is an order of magnitude larger in both Florida and Hawaii than it is in Colorado. To give a similar comparison at the low-frequency end (i.e., from 1 cycle per year to 1 cycle per day), figure 8 shows power spectra of surface refractive index variations in Colorado, Florida, and Hawaii. The Colorado spectrum lies between those of Florida and Hawaii over most of the frequency range except in the vicinity of 1 cycle per day, where the variation in Colorado is relatively larger than at the other locations. Hence it would appear that the rms variation estimates derived from these data lie within a factor of 3 of the values that would have been obtained if the same experiment had been performed in Florida or Hawaii.

\subsection{A Note Regarding "RMS Variations" and Total RMS Error}

It should be emphasized that all rms variations estimated by integration of power spectra are measures of the fluctuations about mean values; i.e., they are standard deviations. (This is true even in the case of rms variations estimated from power spectra that have been extrapolated to zero frequency. In this case the mean value should be regarded as coming from a sample of infinite length.) The total rms error, on the other hand, would be a measure of the fluctuation of a variable about its true value, and would include not only the rms variation about the mean, but also any biases or constant errors which would cause the mean to differ from the true value. No absolute range or range difference measurements were made in this experiment, so it is not possible to estimate bias errors from these data. An important source of bias in estimating the true range and range difference is the possible failure of the actual refractive index structure to conform to the model used to correct range and range difference data with refractivity data. However, a proposed atmospheric model has been developed at the National Bureau of Standards and estimates given of its reliability [Bean and Thayer, 1963; Bean and Thayer, 1959].

\section{References}

Barton, D. K. (April 1963), Reasons for the failure of radio interferometers to achieve their expected accuracy, Proc. IEEE 51, No. 4, 626-627.

Bean, B. R., and G. D. Thayer (May 1959), Models of the atmospheric radio refractive index, Proc. IRE 47, No. 5 $740-755$

Bean, B. R., and G. D. Thayer (May-June 1963), Comparison of observed atmospheric radio refraction effects with values predicted through the use of surface weather observations, J. Res. NBS 6rD (Radio Prop.), No. 3, 273-285.

Thompson, M. C., Jr., H. B. Janes, and A. W. Kirkpatrick (Jan. 1960), An analysis of time variations in tropospheric refractive index and apparent radio-path length, J. Geophys. Res. 65, No. 1, 193-201.

Thompson, M. C., Jr., H. B. Janes and F. E. Freethey (Feb. 1960), Atmospheric limitations on electronic distancemeasuring equipment, J. Geophys. Res. 65, No. 2, 389-393.

Thompson, M. C., Jr., and H. B. Janes (July-Aug. 1959), Measurements of phase stability over a low-level tropospheric path, J. Res. NBS 63D (Radio Prop.), No. 1, 45-51.

Thompson, M. C., Jr., and M. J. Vetter (Feb. 1958), Single path phase measuring system for three-centimeter radio waves, Rev. Sci. Instr. 29, No. 2, 148-150.

Thompson, M. C., Jr., and H. B. Janes (1964), Radio path length stability of ground-to-ground microwave links (to be published).

(Paper 68D11-427) 